\title{
The Pinwheel Sign: Artifact from Head Rotation During SPECT Acquisition for Dopamine Transporter Imaging
}

\author{
Amy K. Janicek ${ }^{1}$, Ryan J. Avery ${ }^{1}$, and Phillip H. Kuo ${ }^{1,2}$ \\ ${ }^{1}$ Department of Medical Imaging, University of Arizona, Tucson, Arizona; and ${ }^{2}$ Departments of Medicine and Biomedical \\ Engineering, University of Arizona, Tucson, Arizona
}

This case study illustrates the pattern and significance of the pinwheel sign, a SPECT artifact from rotational head motion that can complicate the diagnosis of Parkinson disease. Monitoring the patient during image acquisition is crucial since rotational motion artifacts are difficult to identify on raw or processed images and may lead to incorrect interpretation of findings.

Key Words: neurology; quality assurance; SPECT; DAT scan; Parkinson's disease; artifact

J Nucl Med Technol 2014; 42:75-76

DOI: 10.2967/jnmt.113.126078

D

iagnosis of Parkinson disease can be complicated in patients with preexisting neurologic conditions such as essential tremor or drug-induced parkinsonism. In such situations, dopamine transporter imaging improves diagnostic accuracy and optimization of therapy (1). The accuracy of dopamine transporter imaging relies on quality control at all stages in the imaging chain, starting with initial positioning (2). Motion artifacts may lead to incorrect visual reads and may degrade the utility of quantification programs for computer-aided diagnosis (3). Here, we describe the pattern and significance of the pinwheel sign, an artifact from rotational head motion.

\section{CASE STUDY}

A 73-y-old man with Asperger syndrome developed rest and action tremors that interfered with writing and brushing teeth. Propranolol helped to control the action tremor but not the rest tremor. The patient also took valproic acid, which has a potential side effect of tremor.

The neurologist ordered dopamine transporter imaging to differentiate Parkinson disease from essential tremor or druginduced parkinsonism. Two nuclear medicine physicians

Received May 13, 2013; revision accepted Oct. 10, 2013.

For correspondence or reprints contact: Phillip H. Kuo, Department of Medical Imaging, University of Arizona, 1501 N. Campbell Ave, P.O. Box 245067, Tucson, AZ 85724-5067.

E-mail: pkuo@radiology.arizona.edu

Published online Jan. 30, 2014.

COPYRIGHT (c) 2014 by the Society of Nuclear Medicine and Molecular Imaging, Inc.

found the initial scan difficult to evaluate. Both readers inclined to interpret the scan findings as abnormal because of decreased activity in both putamina (worse on the right) (Fig. 1).

However, the technologist noted that the patient had turned his head to the left approximately halfway through the acquisition. The technologist and physician reviewed the sinograms and projection data in cine mode and could not detect the rotary motion. Because the patient could not tolerate a full repeated examination, the acquisition was repeated in half the time and with the head secured to prevent motion.

The 30-min initial acquisition collected 1,740,000 counts, and the 15-min repeated acquisition collected 834,000 counts. The repeated acquisition was considered adequate because more than 1,000,000 counts would have been collected if the study had been classically normal (4). Technologists carefully observed the patient to confirm no motion. Both readers found the repeated scan also difficult to interpret (Fig. 1C). It demonstrated improvement of the anteroposterior orientation of the basal ganglia and unexpected normalization of the right putamen. The first reader again inclined to interpret the findings as abnormal because of decreased activity in the left putamen, but the second reader inclined to interpret the findings as normal because of the imperfect but relatively comma-shaped configuration of the striata. The final diagnosis is being determined through continuing long-term clinical follow-up.

The repeated imaging revealed that leftward rotation of the head caused blurring of the activity in the left caudate head posteriorly, thereby falsely adding activity to the region of the left putamen. Likewise, blurring of the activity in the right putamen anteriorly gave a falsely abnormal right putamen (Fig. 2). The pinwheel analogy was selected because it conjures a mental image of rotary motion with subsequent blurring.

\section{DISCUSSION}

Routinely, technologists assess for motion before submission of images. In myocardial perfusion imaging, technologists examine the cine raw images and sinograms. Physicians can detect motion artifacts on postprocessed images by, for example, the hurricane sign, in which shortaxis slices of the heart resemble the National Weather 


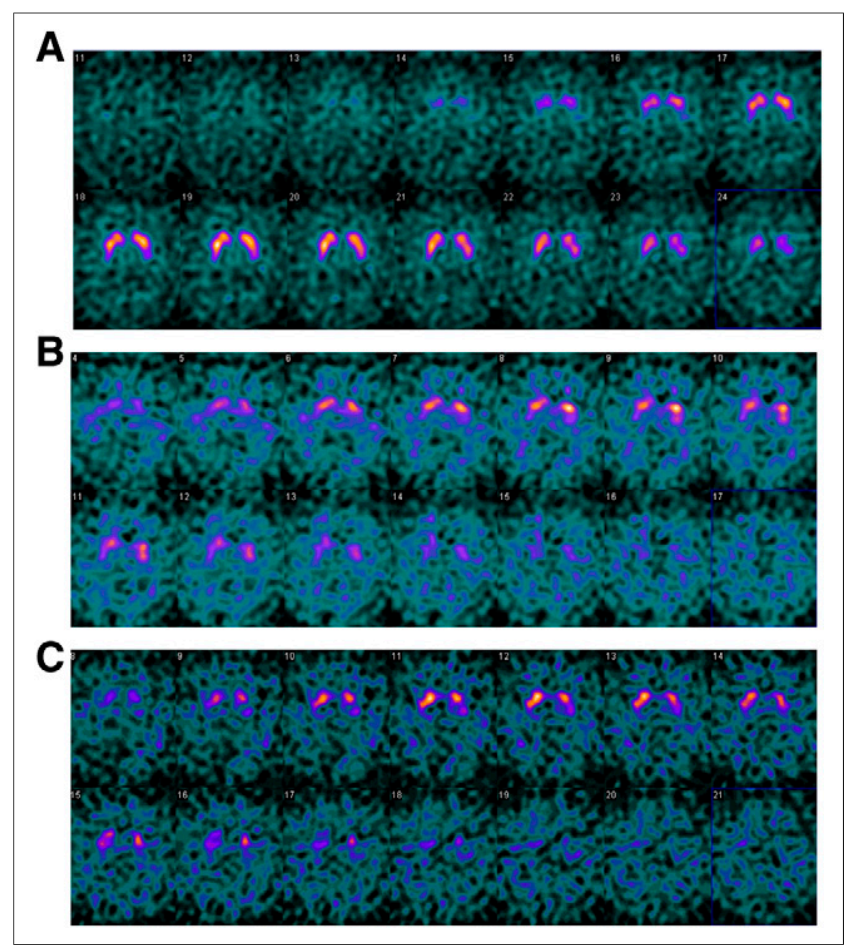

FIGURE 1. (A) Representative ${ }^{123}$-ioflupane scan with normal findings demonstrates properly aligned striata with comma configurations. Dual-head camera set for circular orbit with $13-\mathrm{cm}$ radius acquired data at rate of $30 \mathrm{~s} /$ frame at $3^{\circ}$ per stop for $30 \mathrm{~min}$. Postprocessing reconstruction was performed using filtered backprojection with Butterworth filter with cutoff of 0.6 and order of 8 and no attenuation correction. Axial images have slice thickness of $2.5 \mathrm{~mm}$. (B) Initial imaging was compromised by rotation of head leftward during acquisition. Before injection of 225.7 MBq (6.1 mCi) of ${ }^{123}$ /-ioflupane, patient drank 4 drops of Lugol solution. (C) Imaging was repeated without motion. Standard reconstruction algorithm was same as used for initial scan.

Service symbol for a hurricane (5). Motion artifacts are surprisingly difficult to detect on raw and processed dopamine transporter images, unlike other SPECT images.

\section{CONCLUSION}

Monitoring the patient during SPECT acquisition is crucial because rotational motion artifacts are difficult to

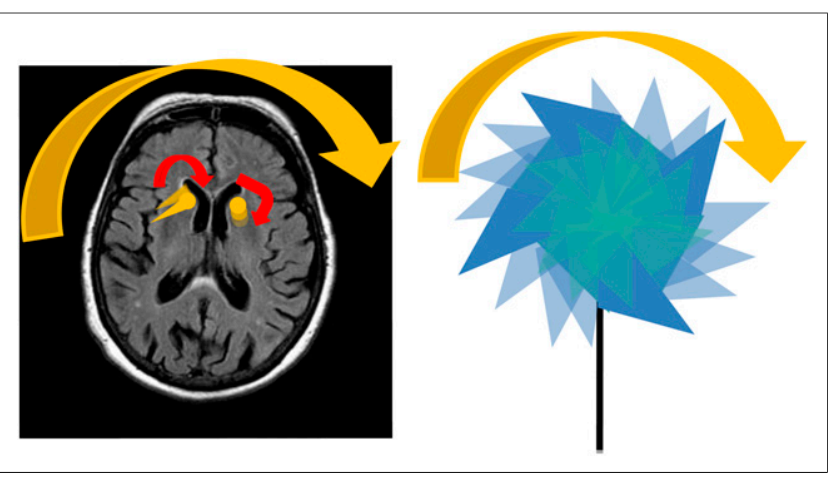

FIGURE 2. (Left) Graphic depicting leftward rotation of caudate heads and putamen (yellow) overlay on MR image of brain. (Right) Illustration of rotating pinwheel for memorable analogy for rotational motion and blurring.

identify on raw or processed images. Clinical interpretation of dopamine transporter images relies on assessment of the morphology of small structures and is therefore particularly susceptible to motion artifacts. Recognizing the pinwheel sign may aid in detecting this critical artifact.

\section{DISCLOSURE}

Phillip H. Kuo has consulted for and received grants from GE Healthcare. No other potential conflict of interest relevant to this article was reported.

\section{REFERENCES}

1. Kägi G, Bhatia KP, Tolosa E. The role of DAT-SPECT in movement disorders. J Neurol Neurosurg Psychiatry. 2010;81:5-12.

2. Covington MF, McMillan NA, Avery RJ, Kuo PH. The semicolon sign: dopamine transporter (DAT) imaging artifact from head tilt. J Nucl Med Technol. 2013;41:105-107.

3. Kuo PH, Avery R, Krupinski E, et al. Receiver-operator-characteristic analysis of an automated program for analyzing striatal uptake of ${ }^{123}$ I-ioflupane SPECT images: calibration using visual reads. J Nucl Med Technol. 2013; 41:26-31.

4. Djang DS, Janssen M, Bohnen N, et al. SNM practice guideline for dopamine transporter imaging with ${ }^{123}$ I-ioflupane SPECT 1.0. J Nucl Med. 2012;53: 154-163.

5. Sorrell V, Figueroa B, Hansen CL. The "hurricane sign": evidence of patient motion artifact on cardiac single-photon emission computed tomographic imaging. J Nucl Cardiol. 1996;3:86-88. 